
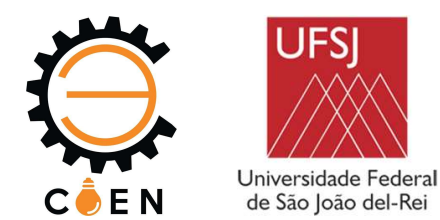

\title{
DEGRADAÇÃO E FORMAÇÃO DE PRODUTOS DE CORROSÃO NO AÇO API X65 EXPOSTO AOS MECANISMOS DE SWEET E SOUR CORROSION
}

Souza, Rhuãn Costa (1) (rhuanmecufsj@gmail.com), Santos, Bernardo Augusto Farah (1) (bernardo.a.fs@hotmail.com), Sabará, Caio Vinícius Leão (1) (caio.leao@oi.com.br), Santos, Luan Carrera (1) (luancarrera777@gmail.com), Prachedes, Lucas Nascimento Santana(1) (lucasntst@gmail.com), Simões,

Thiago Araújo (2) (thiagoasimoes@gmail.com), Oliveira, Jefferson Rodrigues (3) (jefferson.rodrigues@petrobras.com.br),Vaz, Gustavo Leitão (3) (gustavovaz@petrobras.com.br), Bueno, Alysson Helton Santos (1) (alyssonbueno@ufsj.edu.br)

(1) Universidade Federal de São João del Rei (UFSJ) - Dep. de eng. Mec.- 170, Centro, São João del-Rei, MG.

(2) Universidade Federal da Paraíba (UFPB) - Cidade Universitária, João Pessoa, PB.

(3) Centro de Pesquisa da Petrobras (CENPES) - Av. Horácio Macedo, 950 - Cidade Universitária RJ.

RESUMO: O objetivo deste trabalho foi estudar o comportamento eletroquímico e a formação dos produtos de corrosão do aço carbono API X65 quando exposto a sweet e a sour corrosion simultaneamente, conhecendo o caráter competitivo da formação do $\mathrm{FeS}$ e $\mathrm{FeCO}_{3}$. Devido à extrema toxidade e dificuldade de se trabalhar com o $\mathrm{H}_{2} \mathrm{~S}$, a utilização do tiossulfato de sódio se mostra uma excelente alternativa para a geração de $\mathrm{H}_{2} \mathrm{~S}$ na interface metal/solução. Ensaios de EIE e Perda de massa, foram realizados em uma autoclave com pressão parcial de 5 bar de $\mathrm{CO}_{2}$, a 25, 90 e $120^{\circ} \mathrm{C}$ com e sem a presença de $\mathrm{H}_{2} \mathrm{~S}$. Os produtos formados foram caracterizados por meio de MEV e DRX. Nas condições sem $\mathrm{H}_{2} \mathrm{~S}$, a $25^{\circ} \mathrm{C}$ nenhum filme se formou sobre as amostras, a $90^{\circ} \mathrm{C}$ uma película porosa de $\mathrm{FeCO}_{3}$ se formou sobre o material porém os resultados de EIE indicaram uma baixa capacidade protetiva. Já a $120^{\circ} \mathrm{C}$ o filme de $\mathrm{FeCO}_{3}$ se formou mais estável e denso, reduzindo os processos corrosivos. A adição do ion tiossulfato se mostrou eficiente para simular os efeitos do $\mathrm{H}_{2} \mathrm{~S}$, já que foram identificados produtos aderentes da sour corrosion. Os filmes de FeS formados reduziram os efeitos corrosivos do $\mathrm{CO} 2$ e dificultaram a precipitação de $\mathrm{FeCO}_{3}$ no material. A competição de formação das películas foi mais intensa a $120^{\circ} \mathrm{C}$, onde o $\mathrm{FeCO}_{3}$ e o $\mathrm{FeS}$ encontraram condições estáveis de formação.

PALAVRAS-CHAVE: sweet/sour corrosion, EIE, ensaio de perda de massa, tiossulfato de sódio.

\section{INTRODUÇÃO}

As indústrias de óleo e gás têm se deparado com diferentes condições cada vez mais agressivas para as estruturas e tubulações metálicas no ponto de vista da corrosão. Entre os diversos contaminantes corrosivos presentes destacam-se o $\mathrm{CO}_{2}$ e o $\mathrm{H}_{2} \mathrm{~S}$. Os processos corrosivos relacionados a esses compostos, sweet e sour corrosion respectivamente, devem ser detalhadamente estudados a fim de se reduzir ao máximo os prejuízos causados no setor.

$\mathrm{O}$ gás $\mathrm{CO}_{2}$ seco em contato com as superfícies metálicas não é agressivo, porém quando se dissolve no meio aquoso no interior dos poços, se dissocia e forma ácido carbônico $\left(\mathrm{H}_{2} \mathrm{CO}_{3}\right)$ e produtos intermediários segundo as reações (ABELEV et al., 2009, BARKI, 2011):

$$
\begin{gathered}
\mathrm{H}_{2} \mathrm{O}+\mathrm{CO}_{2} \rightarrow \mathrm{H}_{2} \mathrm{CO}_{3} \\
\mathrm{H}_{2} \mathrm{CO}_{3}+2 \mathrm{Fe} \rightarrow \mathrm{Fe}_{2} \mathrm{CO}_{3}+\mathrm{H}_{2}
\end{gathered}
$$

Atingindo-se concentrações elevadas de $\mathrm{CO}_{3}{ }^{2-}$ e $\mathrm{Fe}^{2+}$ pode haver a precipitação do carbonato de ferro na solução ou a formação de uma camada sobre a superfície do material.

$$
\mathrm{Fe}^{2+}+\mathrm{CO}_{3}{ }^{2-} \rightarrow \mathrm{FeCO}_{3}(3) \mathrm{Fe}^{2+}+\mathrm{HCO}_{3}{ }^{-}+\mathrm{e}^{-} \rightarrow \mathrm{FeCO}_{3}+\mathrm{H}
$$




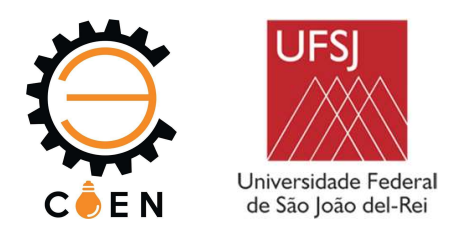

Devido à extrema toxidade e dificuldade de se trabalhar com o gás sulfídrico $\left(\mathrm{H}_{2} \mathrm{~S}\right)$ em laboratório, a utilização do tiossulfato de sódio se mostra uma excelente alternativa para a geração de H2S na interface metal/solução (Kappes et al., 2012; Tsujikawa et al., 1993).

Para Kappes (2012), as formas para que se gere H2S a partir do tiossulfato são:

$$
\begin{gathered}
\mathrm{S}_{2} \mathrm{O}_{3}{ }^{2-}+6 \mathrm{H}^{-}+4 e^{-} \rightarrow 2 \mathrm{~S}^{0}+3 \mathrm{H}_{2} \mathrm{O} \\
2 \mathrm{~S}^{0}+4 \mathrm{H}^{+} \rightarrow+2 \mathrm{H}_{2} \mathrm{~S}
\end{gathered}
$$

Essas reações ocorrem de forma espontânea no potencial de corrosão do aço carbono. Como na maioria das vezes os dutos de extração e transporte de óleo e gás, estão expostos tanto a sweet quanto a sour corrosion e condições de elevas pressões e temperaturas, fatores que influenciam diretamente na formação do $\mathrm{FeCO}_{3}$ e FeS, torna-se importante o estudo da interação desses dois mecanismos corrosivos para a compreensão da formação dos filmes nas diversas condições encontradas in situ.

\section{MATERIAIS E MÉTODOS}

O material utilizado no estudo foi o aço carbono API X65, exposto a meios agressivos contendo $\mathrm{CO}_{2}$ e $\mathrm{H}_{2} \mathrm{~S}$.

As soluções estudadas continham 600 ppm de íons $\mathrm{Cl}^{-}$, e 10-3 mol/L de tiossulfato de sódio. Foi utilizada uma autoclave para submeter as amostras às condições de $25^{\circ} \mathrm{C}, 90^{\circ} \mathrm{C}$ e $120^{\circ} \mathrm{C}$ à 5 bar de $\mathrm{CO}_{2}$.

\section{Preparação das amostras}

Para os ensaios de EIE, os corpos de prova foram usinados de forma cilíndrica com diâmetros de $6 \mathrm{~mm}$ e comprimento de $30 \mathrm{~mm}$ com uma rosca de $1 / 8$ " BSW para a conexão com a haste de contato da autoclave. Os corpos de prova utilizados para a caracterização e perda de massa foram usinados com 20x35x2 mm com furos de $5 \mathrm{~mm}$ de diâmetro próximos a uma das extremidades para auxiliar na fixação.

Todos os corpos de prova foram lixados até a lixa \#600, lavados com água destilada, secos com ar quente e armazenados em um dessecador a vácuo.

\section{Caracterização Metalográfica}

As amostras foram analisadas por microscopia óptica (MO), no microscópio óptico Olympus - BX51, microscopia eletrônica de varredura (MEV), no equipamento HITACHI - TM 3000. Para a caracterização da microestrutura, as amostras foram atacadas com Nital $2 \%$ por $10 \mathrm{~s}$.

Para cada condição estudada, as amostras foram analisadas por MEV e Difratometria de Raios-X (DRX), no aparelho Bruker - D8 Advance com $2 \Theta$ de 15 a $80^{\circ}$.

\section{Ensaios de EIE}

Todos os testes foram realizados no interior da autoclave segundo o esquema representado na Figura 1. Onde a pressão, temperatura e a ausência de oxigênio durante os ensaios eram controlados. Todos os corpos de prova foram colocados ao mesmo tempo no interior da célula 1 (Fig. 1), a solução foi armazenada na célula 2 e o sistema foi fechado para uma desaeração de 4 horas com borbulhamento de $\mathrm{N}_{2}$. 


\section{Seja seu próprio

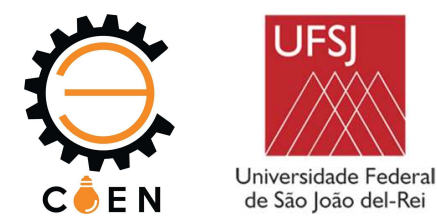

Foi utilizado um potenciostato AUTOLAB: $\mu$ Autolab tipo III/FRA2 e o software NOVA 1.11.2 para a aquisição e tratamento dos resultados.

As medidas foram realizadas, conforme a rotina descrita anteriormente, na faixa de frequências de $10 \mathrm{mHz}$ a $10 \mathrm{KHz}$, com amplitude de $10 \mathrm{mV}$ e 7 medidas por década de frequência.

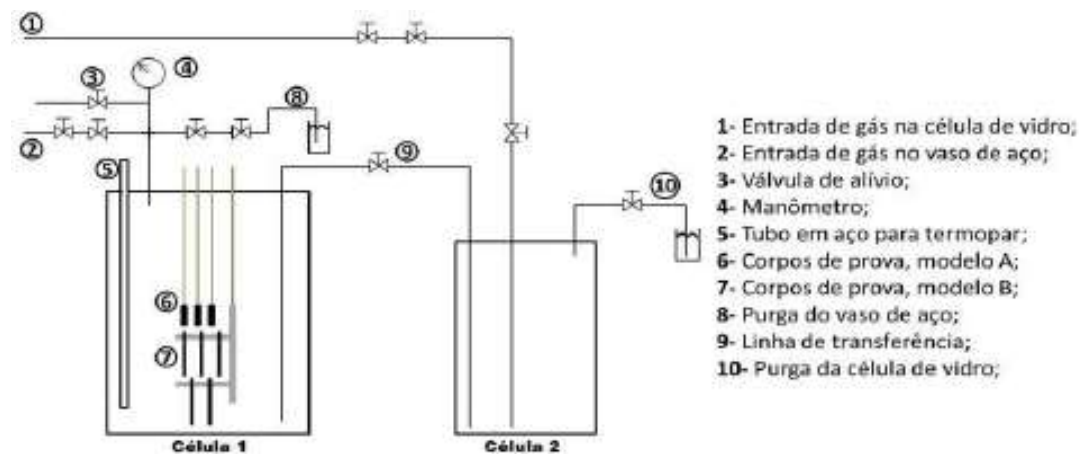

Figura 1: Esquema da Autoclave utilizada nos ensaios.

\section{Teste de Imersão e perda de massa}

Após $48 \mathrm{~h}$ de imersão as amostras foram retiradas da autoclave, lavadas com água destilada, limpas com acetona, secas com ar quente e pesadas, imediatamente, em uma balança de precisão. As amostras passaram, então, por um processo de decapagem com pasta abrasiva e foram pesadas novamente. Os cálculos da taxa de corrosão foram realizados de acordo com a norma ASTM G31.

\section{RESULTADOS}

\section{Caracterização do material}

A microestrutura do material é composta de grãos de ferrita e perlita em uma distribuição homogênea (Figura 2-A). As colônias de perlita fina estão localizadas preferencialmente nos contornos de grão de ferrita, que apresentam uma irregularidade nos tamanhos (Figura 2-B).

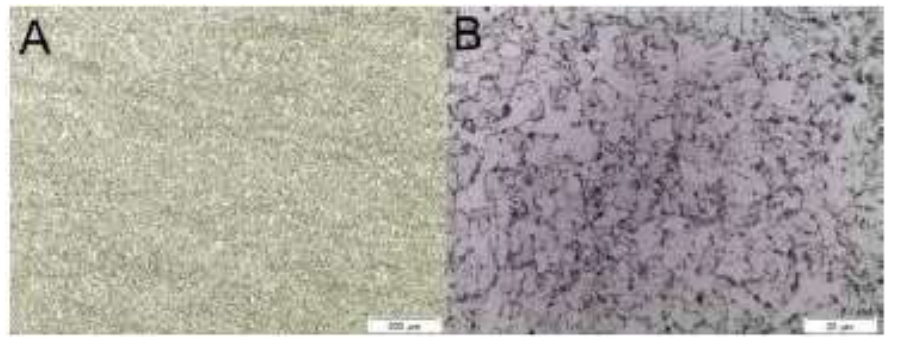

Figura 2: Microscopia ótica do aço API X65, aumentos (A)100x e (B)1000x.

\section{Espectroscopia de Impedância Eletroquímica (EIE)}

Nas curvas de Nyquist (Figura 3), para C1 nota-se a presença de um semicírculo capacitivo em altas frequências e o aparecimento de um segundo arco, de caráter indutivo nas frequências mais baixas. Esse comportamento foi mantido para todos os intervalos de imersão observados no gráfico. 


\section{Seja seu próprio Diferencial}

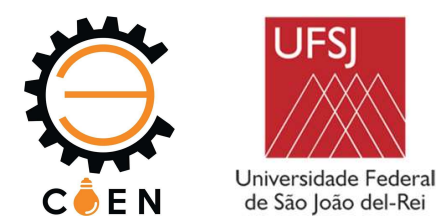

$\mathrm{Na}$ segunda condição (C2), com o aumento da temperatura de $25^{\circ} \mathrm{C}$ para $90^{\circ} \mathrm{C}$, esse comportamento se manteve até aproximadamente 18 horas de imersão, onde possivelmente ocorre uma alteração na interface metal/solução, evidenciada pelo surgimento de um terceiro semicírculo, capacitivo, nas menores frequências.

$\mathrm{Na} \mathrm{C} 3$, com a temperatura de $120^{\circ} \mathrm{C}$ a existência dos mesmos três semicírculos se manteve. Observa-se um aumento significativo do segundo semicírculo capacitivo formado nas frequências mais baixas, que pode estar relacionado com a formação de produtos de corrosão, possivelmente indicando a formação da película de $\mathrm{FeCO}_{3}$ e só aparece após as $12 \mathrm{~h}$ de imersão.

Com a adição de tiossulfato de sódio, as curvas para C4, C5 e C6 apresentaram comportamentos distintos. Os diagramas de Nyquist da $\mathrm{C} 4$, a $25^{\circ} \mathrm{C}$, apresentaram somente um semicírculo capacitivo que aumentou com o aumento do tempo de imersão (Figura 3).

As condições C5 e C6 mostram dois semicírculos capacitivos, o primeiro formado em altas frequências e menor e um segundo arco incompleto, que aparece desde a primeira hora de imersão nas frequências intermediárias a baixas. Esse comportamento indica uma redução na capacitância da dupla camada, um aumento da transferência de carga e uma possível posterior formação de camada aderente dos produtos de corrosão.

É possível observar que com a adição do tiossulfato e consequente geração de $\mathrm{H}_{2} \mathrm{~S}$, houve o desaparecimento do arco indutivo nos diagramas de Nyquist para todos os tempos de imersão apresentados (Figura 3).
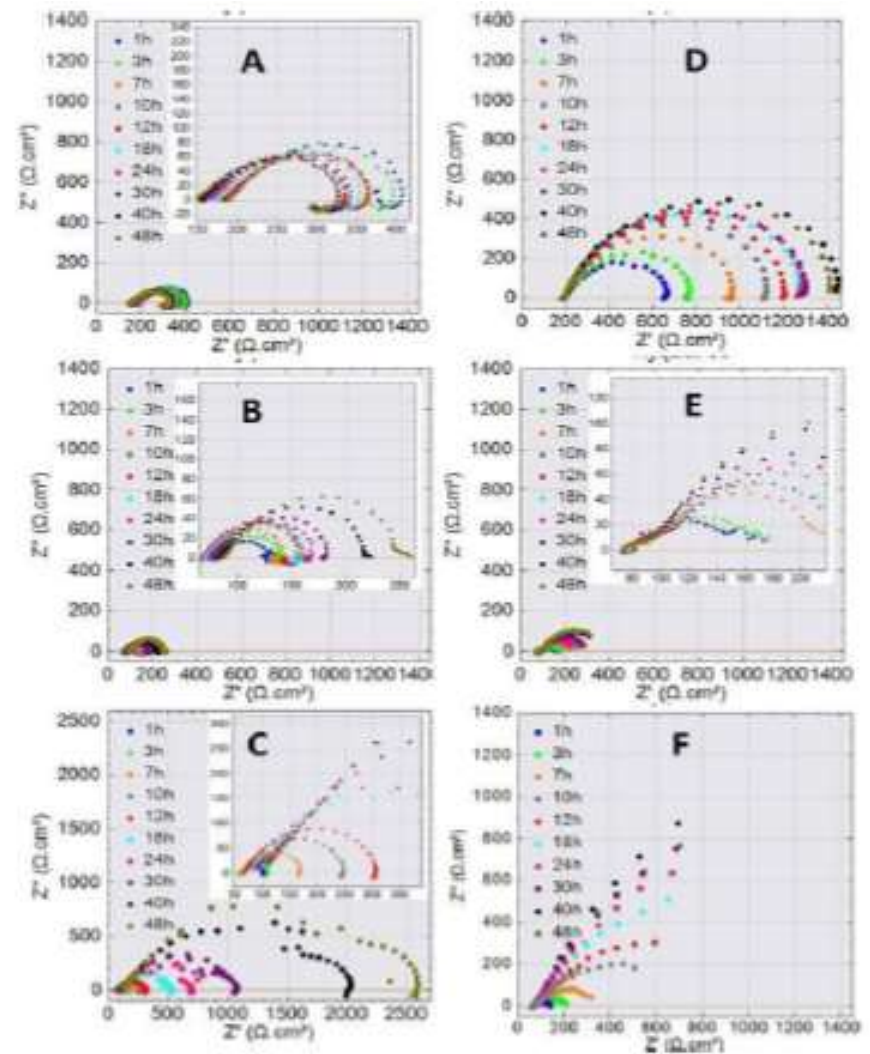

Figura 3: Nyquist para as 6 condições. $\mathrm{C} 1$ a C6, de A a F respectivamente. 


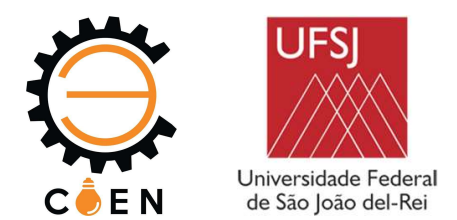

$\mathrm{O}$ modulo de $\mathrm{Z}$, calculado a partir dos valores de $\mathrm{Z}$ real e imaginário, variou significativamente com as condições e com o tempo de imersão. A Figura 4 mostra a evolução do valor de $|Z|$ na última frequência avaliada, da primeira hora para a última hora de imersão nas 6 condições. $\mathrm{C} 1$ foi a única a apresentar valores negativos para a variação $\Delta|\mathrm{Z}|$ ao longo do tempo de imersão. $\mathrm{C} 3$ e C6, ambas a $120^{\circ} \mathrm{C}$, foram as que apresentaram maiores variações nos valores de $|\mathrm{Z}|$.

C4 apresentou resultados contrários ao que se esperava antes dos testes. Visto que $\mathrm{C} 1$, também a $25^{\circ} \mathrm{C}$, apresentou dissolução ativa da superfície, o aumento dos arcos capacitivos (Figura 3) e o aumento no valor de $|\mathrm{Z}|$, não eram esperados.

$\mathrm{C} 2$ e $\mathrm{C} 5\left(90^{\circ} \mathrm{C}\right.$, sem e com $\left.\mathrm{H}_{2} \mathrm{~S}\right)$ apresentaram comportamentos e valores semelhantes, os produtos de corrosão formados apresentaram pouca capacidade de retardar os mecanismos corrosivos, consequentemente o módulo da impedância apresentou um pequeno aumento durante o período de imersão (Figura 4).

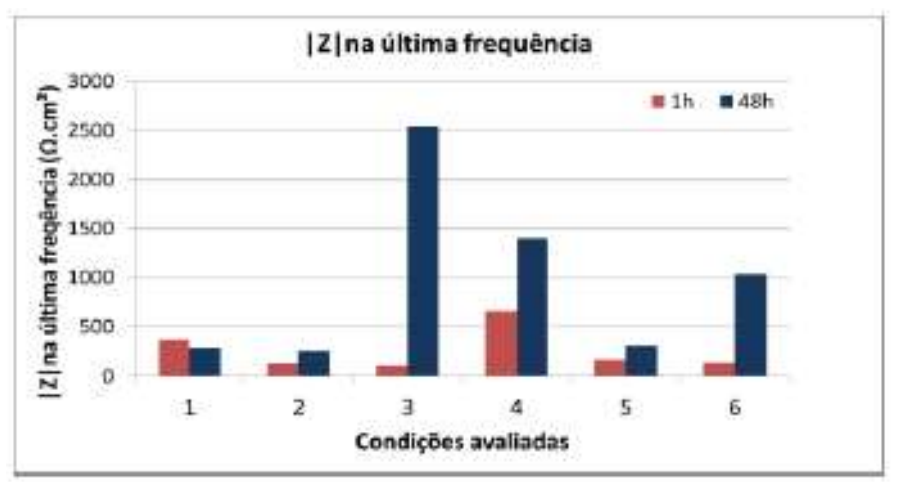

Figura 4: $|Z|$ na última frequência avaliada, da primeira hora para a última hora de imersão.

\section{Testes de perda de massa}

A Figura 5 ilustra o gráfico dos valores de $\Delta \mathrm{m}$ médio, em gramas, antes e depois do processo de decapagem, para todas as condições avaliadas. É possível inferir que as condições onde a diferença entre os valores de $\Delta \mathrm{m}$ antes e após a decapagem são maiores (C2, C3 e C6), são as condições onde houve maior formação de filme na superfície das amostras. A C3 apresentou um ganho de massa em relação a massa inicial ( $\Delta \mathrm{m}$ negativo). Essa condição foi a que apresentou uma camada mais densa de produtos aderentes.

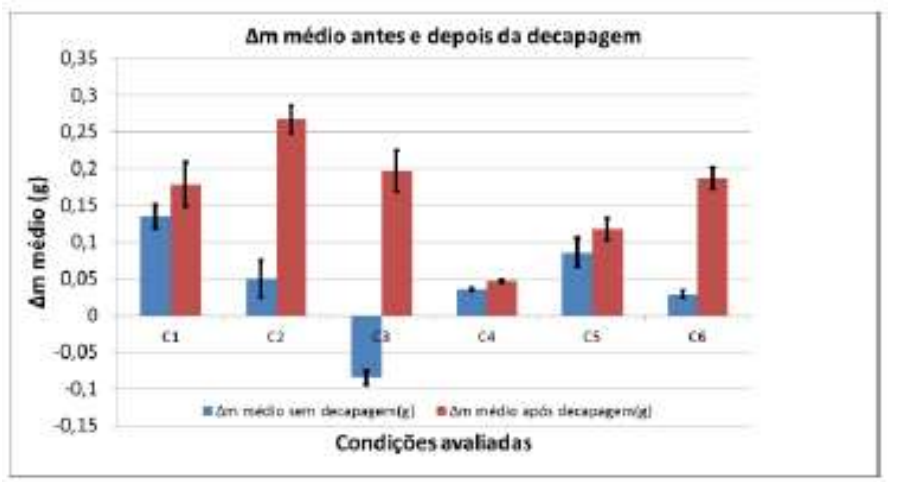

Figura 5: $\Delta \mathrm{m}$ médio, em gramas, antes e depois do processo de decapagem.

IX COEN - Congresso de Engenharias da UFSJ Interconexão. 


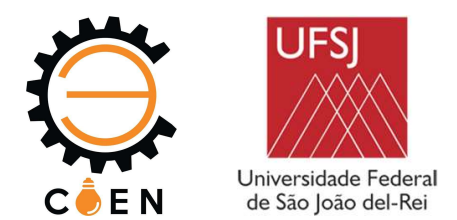

Os valores de $\Delta \mathrm{m}$ após o processo de remoção dos filmes representam a perda de massa real média das amostras, ou seja, a quantidade de material que reagiu com o meio, formando produtos de corrosão aderentes ou não. Através desse valor foi possível calcular a taxa de corrosão para cada condição (Figura 6).

Em C1, C2 e C3 sem a presença do $\mathrm{H}_{2} \mathrm{~S}$, os valores das taxas de corrosão indicam que o aumento da temperatura para $90^{\circ} \mathrm{C}$ favoreceu os processos corrosivos $(2,004 \mathrm{~mm} / \mathrm{y})$, mesmo com a formação de uma possível película na superfície. Já quando a temperatura do teste foi de $120^{\circ} \mathrm{C} o$ valor da taxa de corrosão caiu significativamente $(1,474 \mathrm{~mm} / \mathrm{y})$.

Para C4, C5 e C6, quando houve a geração de $\mathrm{H}_{2} \mathrm{~S}$ na solução, os valores de taxa de corrosão foram menores que sem esse contaminante. $\mathrm{O}$ aumento da temperatura promoveu um grande aumento da taxa de corrosão, como pode ser visto na Figura 6 e. Na literatura esse comportamento, onde o $\mathrm{H} 2 \mathrm{~S}$ e seus produtos reduzem a taxa de corrosão, também é verificado (ABELEV et al., 2009; YIN et al., 2008). A C4, foi a condição que apresentou menor taxa de corrosão entre todas as condições avaliadas.

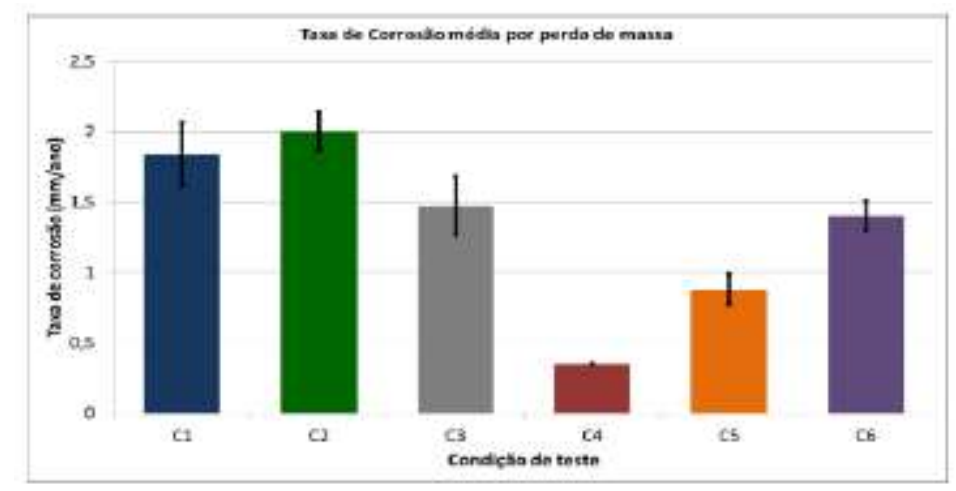

Figura 6: Taxa de corrosão média obtida por perda de massa nas 6 condições estudadas.

\section{Análises dos produtos de corrosão}

A figura 7 apresenta uma amostra sem imersão e uma exposta em cada condição testada.

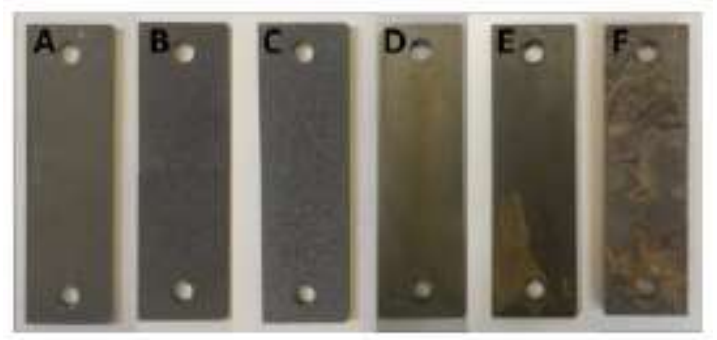

Figura 7: Amostras após o período de imersão nas 6 condições. C1 a C6, de A a F respectivamente.

Os produtos de corrosão aderidos às superfícies, possivelmente, são $\mathrm{FeCO}_{3}$ e $\mathrm{FeS}$ e suas variações. A Figura 8 apresenta um conjunto de imagens de C1 a C6 com ampliações de 400x. 


\section{Seja seu próprio Diferencial}
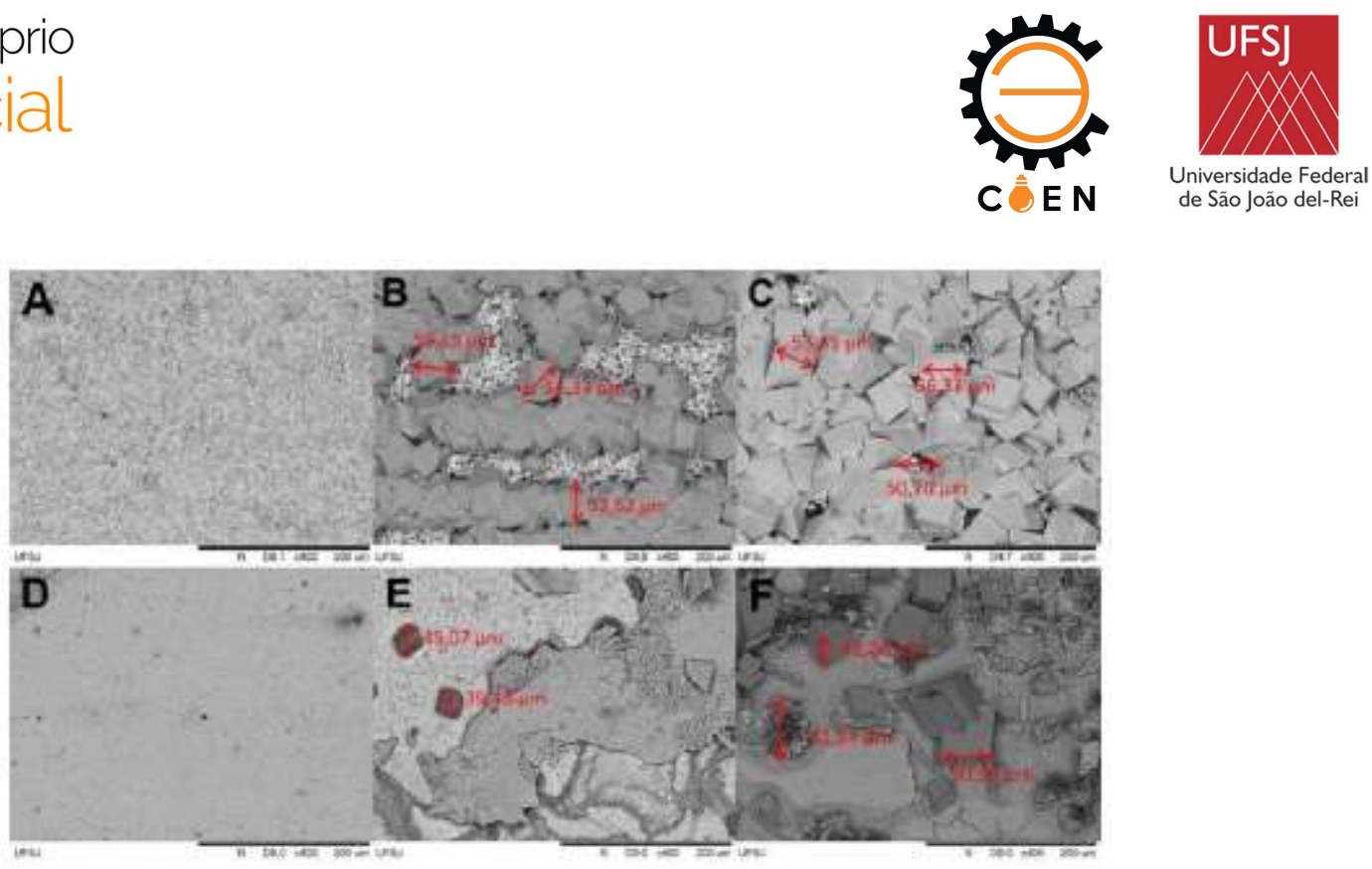

Figura 8: Microscopias Eletrônicas de Varredura das amostras expostas às 6 condições estudadas, aumento de 400x, C1 a C6, de A a F respectivamente.

A amostra da $\mathrm{C} 1\left(25^{\circ} \mathrm{C}\right.$, sem $\left.\mathrm{H}_{2} \mathrm{~S}\right)$ não apresentou nenhuma formação densa de produtos de corrosão aderente ao material, corroborando com as informações dadas pelos ensaios de perda de massa (Figura 8-A).

Os resultados e gráficos da $\mathrm{C} 2\left(90^{\circ} \mathrm{C}\right.$, sem $\left.\mathrm{H}_{2} \mathrm{~S}\right)$, sugerem a formação de uma película protetora de $\mathrm{FeCO} 3$, que gerou uma redução na taxa de corrosão e uma maior variação de massa após o processo de decapagem. Essa formação é comprovada pela análise de MEV, onde é possível notar a precipitação de cristais arredondados e sobrepostos, dispostos em faixas (Figura 8-B).

A C3 $\left(120^{\circ} \mathrm{C}\right.$, sem $\left.\mathrm{H}_{2} \mathrm{~S}\right)$, apresenta uma formação densa e aderente sobre a superfície do aço, o que também foi sugerido pelos valores de perda de massa. A película formada a $120^{\circ} \mathrm{C}$ apresentou características morfológicas diferentes da anterior. Observa-se a existência de muitos poros ou falhas na camada formada a $90^{\circ} \mathrm{C}$, deixando o substrato de aço ainda exposto e em contato com o meio, como visto na Figura 9. O fato da não cobertura total do aço pela camada de carbonato justifica a alta taxa de corrosão apresentada para $\mathrm{C} 2$.

O filme formado na C3 apresentou uma forma cúbica e uma distribuição de tamanho mais heterogênea. Outra diferença está no grau de recobrimento dessa película, sendo possível observar áreas expostas muito menores da superfície do aço. Essa densidade maior do filme está retratada na redução da taxa de corrosão apresentadas por essa condição. Nota-se a precipitação de cristais muito menores em algumas regiões da amostra (Figura 9), esses cristais mais refinados contribuem para o recobrimento do filme sobre o substrato. 


\section{Seja seu próprio Diferencial}
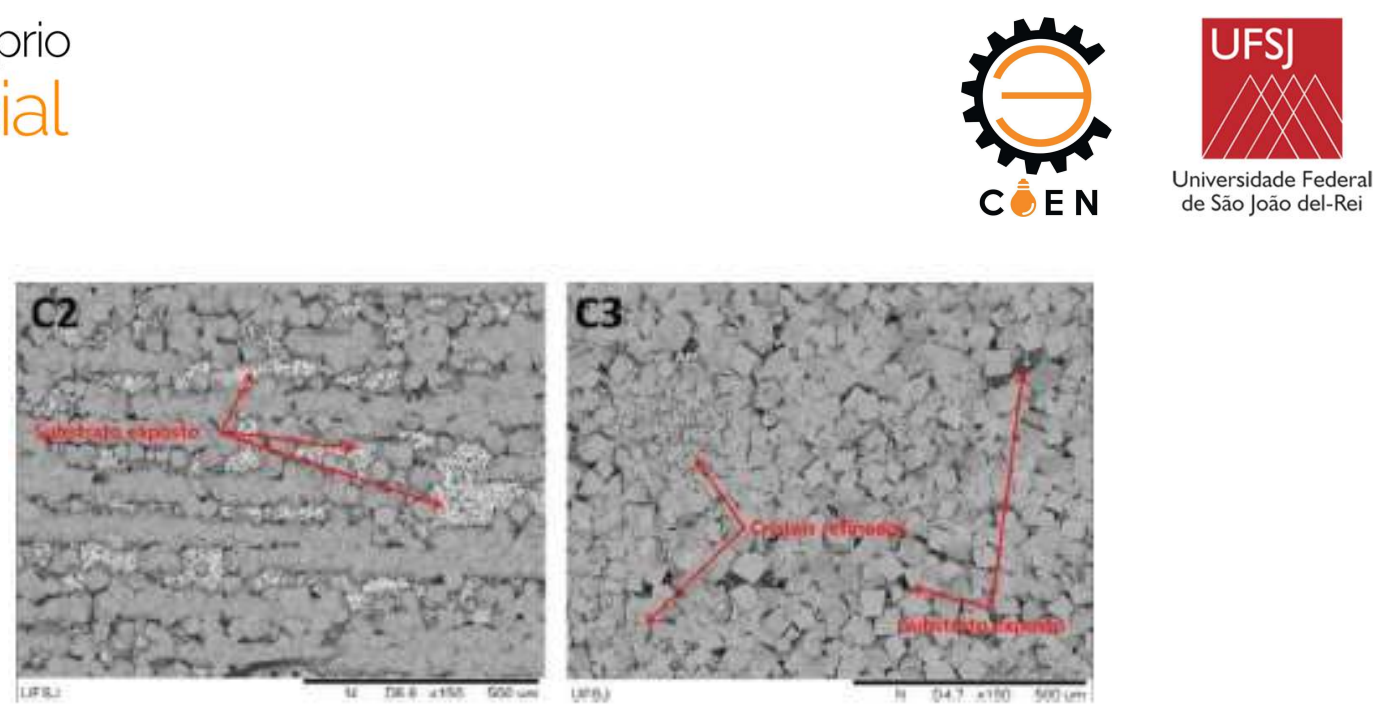

Figura 9: Substrato exposto na amostra da condição 2 e Substrato exposto e Cristais mais finos na amostra da condição 3 , aumento de 150x.

A C4 (Figura 8-D) não demonstra a formação de produtos aderentes durante o período de imersão. Nessa condição, o filme de sulfeto pode ter se formado imediatamente após o contato do aço com a solução. Esse filme de sulfeto se mostrou altamente instável e não permaneceu aderente à superfície ao final do teste, porém pode ter sido o responsável pela baixa taxa de corrosão apresentada pela amostra na $\mathrm{C} 4$, que pode ser notada pelo estado da superfície da amostra $\mathrm{C} 4$ comparada com a amostra $\mathrm{C} 1$, ambas a $25^{\circ} \mathrm{C}$ e expostas a 5 bar de $\mathrm{CO}_{2}$ (Figura 8-A e D). O filme inicial de FeS pode ter retardado o processo da sour corrosion nessa condição e, assim como na condição 1, não foram encontrados cristais de carbonato sobre a superfície das amostras.

Analisando-se o MEV da amostra da $\mathrm{C} 5\left(90^{\circ} \mathrm{C}\right.$, com $\left.\mathrm{H}_{2} \mathrm{~S}\right)$ (Figura 8-E), nota-se a presença de dois produtos distintos aderidos à superfície, porém há a predominância da formação de uma película escura, que possivelmente seja o FeS. Essa película apresenta-se quebradiça e se desprendendo facilmente do substrato, como é possível visualizar, também, na Figura 8-F. Comparando-se a Figura 8-B e E, fica claro que a formação dos cristais de carbonato foi prejudicada pela adição do $\mathrm{H}_{2} \mathrm{~S}$, o filme de sulfeto de ferro pode ter retardado o processo corrosivo e desfavorecido a liberação das espécies iônicas necessárias para a precipitação do carbonato de ferro. Os cristais encontrados na C5 apresentaram morfologia e tamanho similares aos encontrados na $\mathrm{C} 2$, porém se precipitaram isoladamente e em quantidades muito menores na superfície da amostra como mostra a Figura 10.

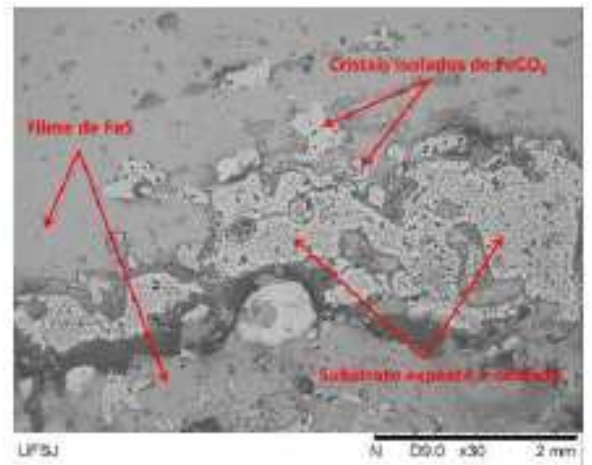

Figura 10: Película descontínua de FeS, Cristais isolados de $\mathrm{FeCO}_{3}$ e substrato exposto na condição 5, aumento de 30x. 


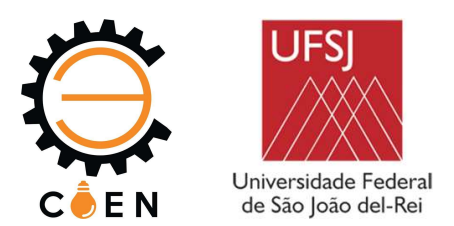

O processo competitivo de formação dos filmes dos produtos de corrosão se mostrou mais aparente nas análises de $\mathrm{MEV}$ da $\mathrm{C} 6\left(120^{\circ} \mathrm{C}, \operatorname{com~}_{2} \mathrm{~S}\right)$, apresentada na Figura 8-F. A película escura presente na $\mathrm{C} 5$ também foi observada na $\mathrm{C} 6$, porém apresentando densidade e estabilidade superiores nos testes a $120^{\circ} \mathrm{C}$. É possível observar na Figura $8-\mathrm{G}$ que a amostra teve praticamente toda a superfície recoberta por produtos de corrosão aderentes e estáveis ao final do teste de imersão.

Além da alteração nas características da película de $\mathrm{FeS}$, os cristais de carbonato sofreram grandes mudanças de morfologia e tamanho. Assim como a alteração notada da C2 para a C3, os cristais da C6 apresentam formatos cúbicos, porém nota-se a formação de produtos de corrosão na forma de aglomerados de lâminas (Figura 11), a condição 6 apresentou, também, mais um produto da sweet corrosion. Os cristais de forma arredondada, similares ao carbonato de ferro formado a $90^{\circ} \mathrm{C}$ (Figura 12), se precipitaram de forma isolada sobre a camada mais densa de FeS.

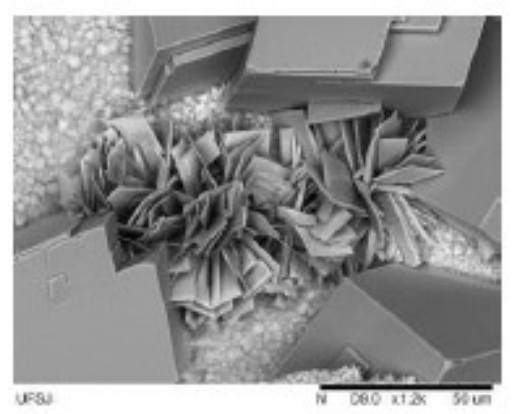

Figura 11: Formação do FeCO3 em lâminas na C6, aumento 1200x.

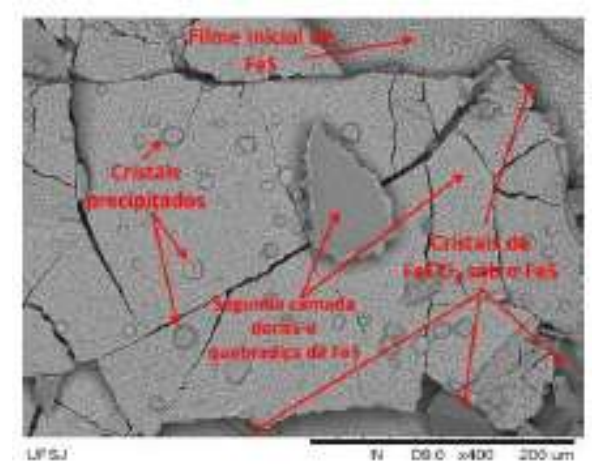

Figura 12: Produtos de corrosão na amostra da condição 6, aumento 400x.

A Figura 13 apresenta os espectros obtidos para as amostras estudadas. Todas as análises foram realizadas com base nas cartas disponíveis no site da AMCSD. 


\section{Seja seu próprio Diferencial}
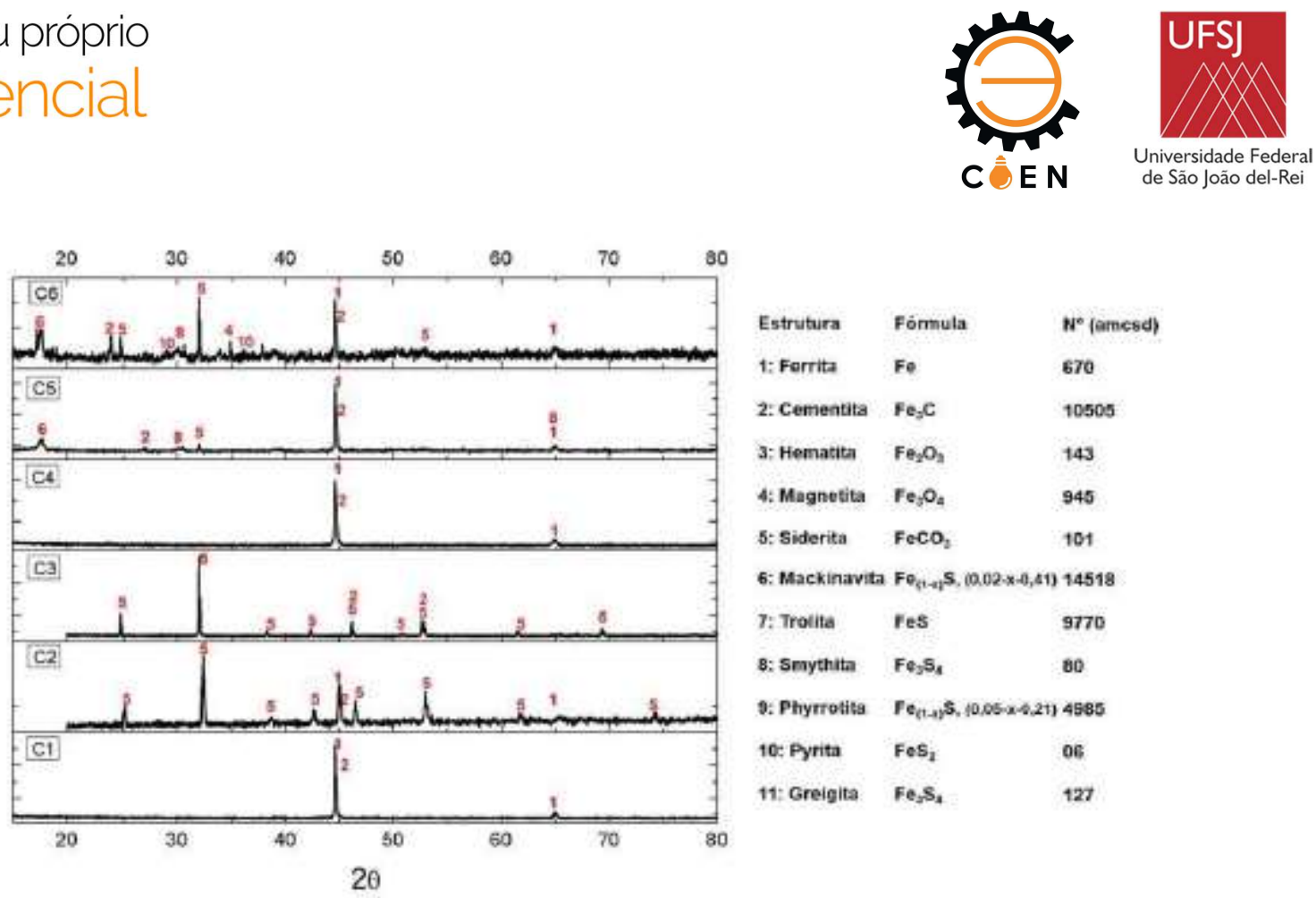

Figura 13: Espectros de difração de Raios-X das amostras expostas às 6 condições.

A amostra $\mathrm{C} 1$ apresentou picos representativos dos compostos Ferrita e Cementita, presentes na microestrutura do aço API X65 analisada na Figura 1 (microscopia ótica do material). Não foi constatado, realmente, a formação de nenhum produto aderente ou óxido na superfície da amostra, o que já havia sido observado nas análises de MEV (Figura 8-A).

Analisando-se o espectro referente à $\mathrm{C} 2$, fica claramente comprovada a precipitação do carbonato de ferro na superfície do corpo de prova. Nota-se, também, que ainda são detectadas as fases ferrita e cementita, esse fato comprova a existência de poros e/ou falhas no filme de $\mathrm{FeCO}_{3}$.

$\mathrm{A}$ amostra $\mathrm{C} 3$ apresentou resultados semelhantes. Um grande número de picos referentes à Siderita, ou carbonato de ferro, $\mathrm{FeCO}_{3}$ pode ser detectado. Porém nos testes a $120^{\circ} \mathrm{C}$ sem $\mathrm{H}_{2} \mathrm{~S}$, a dissolução da ferrita ocorreu de forma mais acentuada, o que favoreceu a formação da película mais densa, observada no MEV (Figura 8-C) e os poros e falhas no filme de carbonato, deixaram exposto um substrato rico em cementita, detectada na espectroscopia dessa condição.

A C4, $\left(25^{\circ} \mathrm{C}\right.$ e $\left.\mathrm{H}_{2} \mathrm{~S}\right)$ não apresentou a formação de nenhum cristal aderente à superfície. A espectroscopia identificou apenas Ferrita e Cementita (Figura 13). Já a C5 $\left(90^{\circ} \mathrm{C} \mathrm{e} \mathrm{H}_{2} \mathrm{~S}\right)$. Além das fases referentes ao substrato do aço, Ferrita e Cementita, a formação de cristais de Siderita, apresentada no MEV de forma isolada e em poucas quantidades (Figura 8-E), também é confirmada pela presença do pico referente a essa fase. A formação da camada escura e quebradiça percebida na Figura 8-E pode ser relacionada com os picos de mackinawita e smitita identificados.

$\mathrm{Na}$ análise de DRX da condição 6 ficou claro a competição de formação dos produtos de corrosão da sweet e sour corrosion. O substrato metálico ainda exposto pode ter sido identificado devido à instabilidade dos filmes de sulfeto à temperatura ambiente. A formação de siderita foi facilmente percebida nos MEVs (Figura 8-F) e comprovada nos espectros (Figura 13), além da formação de um novo composto rico em ferro e oxigênio, a magnetita $\left(\mathrm{Fe}_{3} \mathrm{O}_{4}\right)$.

Provavelmente devido ao aumento da temperatura, além do aumento da intensidade do pico relativo à mackinawita e da smitita, a pirita também foi identificada. 


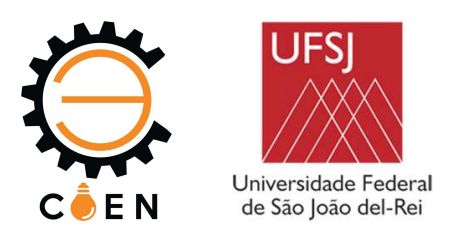

\section{DISCUSSÕES}

\section{Caracterização do material}

O aço API X65 é largamente utilizado na fabricação de dutos para a indústria de óleo e gás. A microestrutura ferrítica/perlítica (Figura 1) encontrada também é destacada por Gao e seus colaboradores (2011).

Zhao e outros (2016b) afirmam que a cementita apresenta um potencial de corrosão mais positivo que a ferrita. Esse efeito galvânico gerado entre a $\mathrm{Fe} 3 \mathrm{C}$ e a $\alpha$-Fe favorece a dissolução inicial das fases ferríticas e consequentemente, a liberação de íons de Fe na solução (FARELAS et al., 2010; ZHAO et al., 2016a). Outra influência da dissolução da ferrita na solução é a geração dos produtos intermediários, como o $\mathrm{FeOH}, \mathrm{FeCO}_{2} \mathrm{OH}$ e $\mathrm{FeHCO}_{3}$, que se adsorvem na superfície das amostras. A alteração de cor observada na Figura 5-B e a variação de massa da amostra C1 (Figura 3) podem ser atribuídas a esses produtos.

\section{Influência da temperatura}

A temperatura é o fator mais impactante nas reações e nos mecanismos da sweet e sour corrosion. Ela tem um impacto substancial na solubilidade de $\mathrm{CO}_{2}$ nas soluções aquosas, alterando a ionização do ácido carbônico. Esse efeito altera a concentração das espécies iônicas presentes na solução, o que pode promover uma modificação no pH do meio (ELGADDAFI et al., 2015; SHI et al., 2016; NING et al., 2015).

A temperatura reduziu o diâmetro do primeiro arco capacitivo formado a altas frequências (resistência a transferência de carga e a capacitância da dupla camada elétrica), tanto para as condições sem H2S quanto para as condições onde o contaminante foi adicionado (Figura 3). Ezuber (2009) relata que a redução da resistência à polarização está relacionada com o aumento da temperatura. $\mathrm{O}$ efeito da temperatura, sobre a dissociação do $\mathrm{CO}_{2}$ na solução, altera a constante de equilíbrio dessas reações, refletindo no aumento das quantidades de $\mathrm{H}^{+}$e $\mathrm{HCO}_{3}$ - no meio.

Observando os resultados das condições C1, C2 e C3 (Apenas CO2), nota-se que o aumento da temperatura favoreceu a precipitação (Figura 8-A,B,C) e a capacidade protetiva do filme de $\mathrm{FeCO}_{3}$, como também encontrado por Tanupabrungsun (2013). A explicação para a maior facilidade e capacidade protetiva do $\mathrm{FeCO}_{3}$ com o aumento da temperatura está na baixa solubilidade do carbonato em solução, quando se tem temperaturas mais elevadas (Elgaddafi et al.,2015).

Os resultados com $\mathrm{H}_{2} \mathrm{~S}$ também se apresentam de acordo com a literatura. Zheng e outros (2015) afirmam que o aumento da temperatura auxilia a formação dos filmes de sulfeto de ferro acelerando a cinética de precipitação, e os filmes de sulfeto podem formar camadas porosas acelerando o processo corrosivo pelo mecanismo de par galvânico. Essa informação corrobora com os resultados apresentados na C4 e também, por Lee (2004), que relata que pequenas concentrações de $\mathrm{H}_{2} \mathrm{~S}$ podem reduzir a taxa de corrosão à temperatura ambiente.

Os espectros de Raios-X apresentados na Figura 13-D, E, F para as condições C4, C5 e C6 confirmam as informações discutidas na literatura. Após a adsorção dos produtos da dissolução do H2S na superfície do aço, o primeiro filme que se forma é a mackinawita, essa fase é metaestável, se forma em baixas temperaturas e é relatada como a principal componente dos filmes de sulfeto (K.-L. J. Lee, 2004). A corrosão do aço carbono pelo sulfeto de hidrogênio em um meio aquoso a baixas 

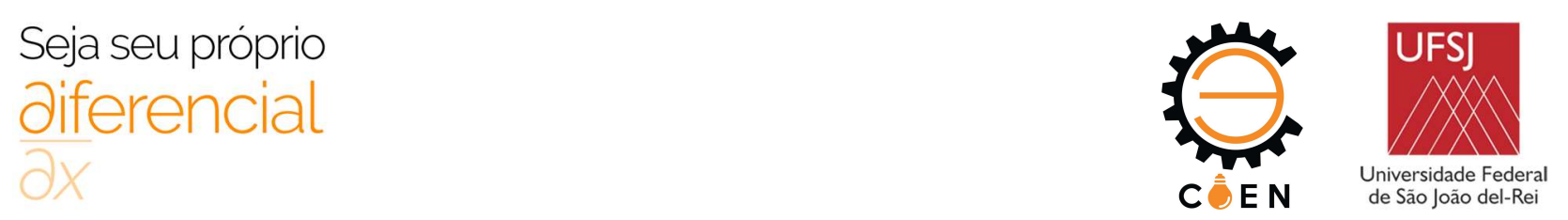

temperaturas envolve, então, na maioria das vezes, a formação de mackinawita que se apresenta quebradiça e facilmente se desprende do substrato (Ezuber, 2009; Ning et al., 2015; Shi et al., 2016).

A temperatura, então, além de favorecer a formação do carbonato e dos sulfetos, alterou a morfologia dos cristais precipitados, isso pode ser observado comparando-se as análises de MEV tanto da $\mathrm{C} 2$ com a C3 quanto as $\mathrm{C} 5$ com a C6, (Figura 8).

\section{Influência do tempo de imersão}

De forma geral, com exceção da $\mathrm{C}$ 1, quanto maior o tempo de imersão, maior a resistência ao processo corrosivo, e maior a quantidade de produtos aderidos à superfície. Zhang e outros (2012), também encontraram comportamento similar.

Encontra-se na literatura (Cardoso, 2012; Farelas et al., 2010; K. Lee, 2004), a relação do arco capacitivo presente nas frequências mais elevadas dos diagramas de Nyquist com a capacitância da dupla camada elétrica formada entre o metal e o eletrólito, assim como indicam a resistência à transferência de carga. A presença de um arco indutivo formado em baixas frequências indicou um processo de adsorção de produtos intermediários da dissolução do ferro $\left(\mathrm{FeOH}, \mathrm{FeCO}_{2} \mathrm{OH}\right.$ e $\mathrm{FeHCO}_{3}$ ), nas condições $\mathrm{C} 1, \mathrm{C} 2, \mathrm{C} 3$ e C4 (Figura 3-A, B, C, D). A formação do terceiro semicírculo (capacitivo) em baixas frequências, indica a formação de uma camada aderente de produto de corrosão, nesse caso, a formação do $\mathrm{FeCO}_{3}$ (LEE, 2004a; SUN et al., 2012).

Nos diagramas de Nyquist da $\mathrm{C} 1$ há uma redução do primeiro semicírculo capacitivo, à medida que se aumenta o tempo de imersão, observa-se que o mecanismo de dissolução ativa encontra facilidade para ocorrer, com o aumento do tempo de imersão (Cardoso, 2012; Farelas et al., 2010; Ren et al., 2005). Quanto maior o tempo de contato metal/solução e solução/ $\mathrm{CO}_{2}$, a concentração de $\mathrm{H}_{2} \mathrm{CO}_{3}$ aumenta com a dissolução do gás na solução, com isso ocorre uma aceleração na cinética das reações catódicas gerando mais íons $\mathrm{CO}_{3}{ }^{2-}$. Para que o equilíbrio eletroquímico seja mantido, a reação anódica de dissolução do ferro deve aumentar e liberar mais $\mathrm{Fe}^{2+}$. Com a supersaturação do meio por essas espécies químicas, a precipitação e formação do filme de $\mathrm{FeCO}_{3}$ se torna possível (Sun et al., 2012; Zhang et al., 2012). O intervalo para o início da formação do filme é discutido e está de acordo com diversos autores (Han et al., 2011; Yang et al., 2014).

Nas condições C4, C5 e C6, com a adição do H2S, os processos corrosivos sofreram alterações, porém o efeito do tempo se manteve semelhante, favorecendo a formação dos filmes desulfeto. Uma camada fina de mackinawita se forma inicialmente. Zhao e outros (2016), também afirmam que os produtos da sour corrosion se formam rapidamente e se desenvolvem mais facilmente por causa da cinética das reações envolvendo o Fe e H2S na fase inicial de imersão.

É possível identificar uma sequência de formação nas películas de corrosão aderidas na superfície da amostra da C6 (Figura 8-F). Inicialmente a primeira camada de FeS, formada imediatamente após o contato do substrato com o H2S. Com o aumento do tempo de imersão, os cristais de $\mathrm{FeCO}_{3}$ se precipitaram sobre a camada porosa de $\mathrm{FeS}$ e o substrato do aço. A medida que o H2S foi gerado pela reação do Tiossulfato, a concentração de $\mathrm{S}^{2-}$ da solução aumentou e a nova camada mais densa e os cristais de FeS se precipitaram sobre os carbonatos de ferro. Zhao e seus colaboradores (2016b) encontraram um comportamento semelhante para as películas formadas até as 24 de imersão em meios contendo $\mathrm{H}_{2} \mathrm{~S}$.

\section{Influência da geração de $\mathrm{H}_{2} \mathrm{~S}$}


Tang e seus colaboradores (2010), relatam que o efeito do $\mathrm{H}_{2} \mathrm{~S}$ sobre a corrosão do aço carbono se desenvolve em dois processos simultâneos, um processo de dissolução preferencial dos grãos de ferrita e um processo de corrosão galvânica nesses grãos. O material estudado apresentou uma microestrutura ferrítica/perlítica (Figura 2), uma dissolução anódica ocorre preferencialmente na ferrita. Consequentemente as reações catódicas ocorrem sobre a cementita. Vários autores (Ezuber, 2009; Kappes, 2011; Xia et al., 2015) afirmam que filmes de sulfeto gerados apresentam um potencial catódico com relação ao substrato, podendo ativar e acelerar as reações anódicas.

Zheng e outros, em estudos recentes (2015), identificaram uma significante redução nas reações anódicas e nas densidades de corrente quando o $\mathrm{H} 2 \mathrm{~S}$ foi adicionado. O mecanismo de redução de transferência de carga vem das alterações geradas na dupla camada elétrica, formada na interface metal/solução. A espessura da camada inicial de FeS é reportada na literatura como sendo da mesma ordem de grandeza da distância da dupla camada elétrica (10 a $100 \mathrm{~nm})($ ZHENG, et al., 2015).

A adição de tiossulfato de sódio na solução se mostrou eficiente na substituição do $\mathrm{H}_{2} \mathrm{~S}$ gasoso para simular a sour corrosion, já que foi comprovada a formação dos produtos aderentes ricos em enxofre, característicos desse mecanismo, nas análises de MEV e DRX.

$\mathrm{O} \mathrm{HS}^{-}$e o $\mathrm{S}^{2-}$ se aderem inicialmente durante a imersão porém apresentaram baixa estabilidade ao fim dos testes. Tanto a adsorção das espécies quanto a formação dos diversos tipos de sulfetos de ferro encontrados, agiram retardando e dificultando as reações da sweet corrosion, seja reduzindo a dissolução ativa do substrato ou dificultando a formação da película de $\mathrm{FeCO}_{3}$.

Kappes e seus colaboradores (2011) afirmam que na maioria das vezes, não ocorre a passivação de substratos recobertos por filmes de FeS devido aos defeitos na estrutura das películas formadas, e da tendência quebradiça e rachaduras características na camada de mackinawita formada em meios com $\mathrm{H}_{2} \mathrm{~S}$.

A formação dos filmes de sulfeto em duas camadas foi observada nas amostras da C5 e C6 como visto na Figura 8, esse tipo de formação dos filmes de sulfeto de ferro também foram relatados por (ZHENG, et al., 2015) e outros autores.

\section{CONCLUSÕES}

Os testes realizados ajudaram a entender os mecanismos corrosivos e o processo de formação competitiva de $\mathrm{FeCO}_{3}$ e FeS. Além disso, o tiossulfato como alternativa ao borbulhamento de $\mathrm{H}_{2} \mathrm{~S}$ foi utilizado com sucesso.

Os gráficos de EIE indicaram que as camadas de $\mathrm{FeCO}_{3}$ são formados preferencialmente após um intervalo de Imersão de $12 \mathrm{~h}$ e as camadas de FeS aparecem nos primeiros estágios de teste.

Filmes de carbonato de ferro apresentaram boa capacidade de proteção. Contudo, devido ao tempo de imersão insuficiente, ainda havia regiões de substrato expostos ao processo corrosivo. Os produtos adsorvidos no início do processo corrosivo pelo $\mathrm{H}_{2} \mathrm{~S}$ apresentaram boa capacidade protetora.

Os testes eletroquímicos realizados mostraram que a temperatura é a fator mais impactante nas reações e mecanismos corrosivos. $\mathrm{O}$ aumento da temperatura gera uma mudança na agressividade inicial da solução. No entanto, também muda a cinética de formação dos filmes de sulfeto e carbonato, favorecendo a formação da camada na superfície da amostra.

\section{REFERÊNCIAS}



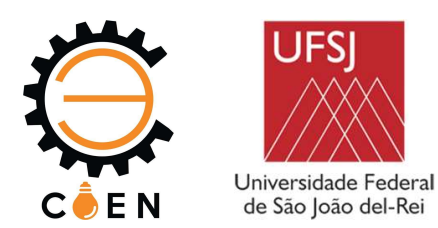

Abelev, E., Sellberg, J., Ramanarayanan, T.A., Bernasek, S.L., 2009. Effect of H2S on Fe corrosion in CO2saturated brine. J. Mater. Sci. 44, 6167-6181. https://doi.org/10.1007/s10853-009-3854-4

Cardoso, A.D.S., 2012. ESTUDO DA CORROSÃO DE LIGAS Fe-Cr-Mo EM ÁGUA ARTIFICIAL DE TANQUE DE ARMAZENAMENTO DE PETRÓLEO SATURADA COM CO2. UNIVERSIDADE FEDERAL DO CEARÁ.

Elgaddafi, R., Naidu, A., Ahmed, R., Shah, S., Hassani, S., Osisanya, S.O., Saasen, A., 2015. Modeling and experimental study of $\mathrm{CO} 2$ corrosion on carbon steel at elevated pressure and temperature. J. Nat. Gas Sci. Eng. 27, 1620-1629. https://doi.org/10.1016/j.jngse.2015.10.034

Ezuber, H.M., 2009. Influence of temperature and thiosulfate on the corrosion behavior of steel in chloride solutions saturated in CO2. Mater. Des. 30, 3420-3427. https://doi.org/10.1016/j.matdes.2009.03.028

Farelas, F., Galicia, M., Brown, B., Nesic, S., Castaneda, H., 2010. Evolution of dissolution processes at the interface of carbon steel corroding in a CO2 environment studied by EIS. Corros. Sci. 52, 509-517. https://doi.org/10.1016/j.corsci.2009.10.007

Han, J., Nešić, S., Yang, Y., Brown, B.N., 2011. Spontaneous passivation observations during scale formation on mild steel in CO2 brines. Electrochim. Acta 56, 5396-5404. https://doi.org/10.1016/j.electacta.2011.03.053

Kappes, M., Frankel, G.S., Sridhar, N., Carranza, R.M., 2012. Reaction Paths of Thiosulfate during Corrosion of Carbon Steel in Acidified Brines. J. Electrochem. Soc. 159, C195. https://doi.org/10.1149/2.085204jes

Kappes, M.A., 2011. Evaluation of thiosulfate as a substitute for hydrogen sulfide in sour corrosion fatigue studies. Diss. - Ohio State Univ. 253.

Lee, K.-L.J., 2004. A MECHANISTIC MODELING OF CO2 CORROSION OF MILD STEEL IN THE PRESENCE OF H2S. Ohio University.

Lee, K., 2004. A mechanistic modeling of CO2 corrosion of mild steel in the presence of H2S. Dissertation. Ning, J., Zheng, Y., Brown, B., Young, D., Nesic, S., Technology, M., 2015. Construction and Verification of Pourbaix Diagrams for Hydrogen Sulfide Corrosion of Mild Steel. Nace Corosion 1-19.

Ren, C., Liu, D., Bai, Z., Li, T., 2005. Corrosion behavior of oil tube steel in simulant solution with hydrogen sulfide and carbon dioxide. Mater. Chem. Phys. 93, 305-309. https://doi.org/10.1016/j.matchemphys.2005.03.010

Shi, F., Zhang, L., Yang, J., Lu, M., Ding, J., Li, H., 2016. Polymorphous FeS corrosion products of pipeline steel under highly sour conditions. Corros. Sci. 102, 103-113. https://doi.org/10.1016/j.corsci.2015.09.024

Sun, J.B., Zhang, G.A., Liu, W., Lu, M.X., 2012. The formation mechanism of corrosion scale and electrochemical characteristic of low alloy steel in carbon dioxide-saturated solution. Corros. Sci. 57, 131138. https://doi.org/10.1016/j.corsci.2011.12.025

Tang, J., Shao, Y., Guo, J., Zhang, T., Meng, G., Wang, F., 2010. The effect of H2S concentration on the corrosion behavior of carbon steel at 90 ??C. Corros. Sci. 52, 2050-2058. https://doi.org/10.1016/j.corsci.2010.02.004

Tanupabrungsun, T., Brown, B., Nesic, S., 2013. Effect of pH on CO2 Corrosion of Mild Steel at Elevated Temperatures. Nace Corosion 1-11.

Tsujikawa, S., Miyasaka, A., Ueda, M., Ando, S., Shibata, T., Haruna, T., Katahira, M., Yamane, Y., Aoki, T., Yamada, T., 1993. Alternative for evaluating sour gas resistance of low-alloy steels and corrosion- resistant alloys. Corrosion 49, 409-419. https://doi.org/10.5006/1.3316068 

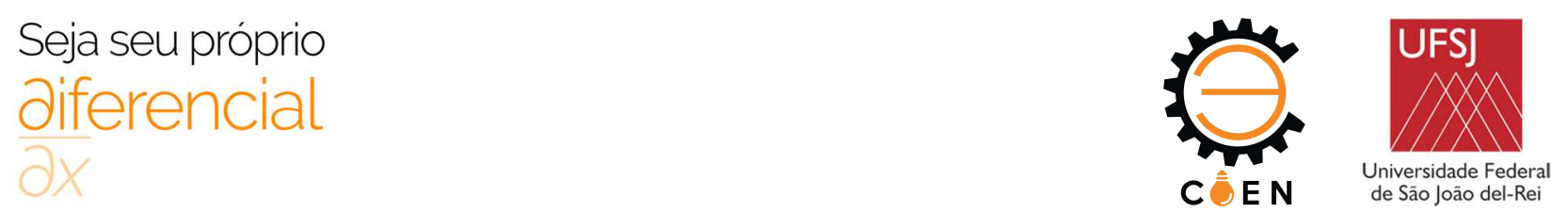

Xia, D.H., Zhu, R.K., Behnamian, Y., Luo, J.L., Lin, C.J., Klimas, S., 2015. Understanding the interaction of thiosulfate with Alloy 800 in aqueous chloride solutions using SECM. J. Electroanal. Chem. 744, 77- 84. https://doi.org/10.1016/j.jelechem.2015.03.006

Yang, D., Rosas, O., Castaneda, H., 2014. FeCO3 layer evolution for API 5L X52 steel in carbon dioxidesaturated $\mathrm{NaCl}$ brine in the presence of 1-decyl-3-methylimidazolium chloride. Corros. Sci. 87, 40-50. https://doi.org/10.1016/j.corsci.2014.05.021

Yougui Zheng, Jing Ning, Bruce Brown, David Young, S.N., 2015. MECHANISTIC STUDY OF THE EFFECT OF IRON SULFIDE LAYERS ON HYDROGEN SULFIDE CORROSION OF CARBON STEEL. Nace Corosion 1-20.

Zhang, G. a., Zeng, Y., Guo, X.P., Jiang, F., Shi, D.Y., Chen, Z.Y., 2012. Electrochemical corrosion behavior of carbon steel under dynamic high pressure $\mathrm{H} 2 \mathrm{~S} / \mathrm{CO} 2$ environment. Corros. Sci. 65, 37-47. https://doi.org/10.1016/j.corsci.2012.08.007

Zhao, W., Zou, Y., Matsuda, K., Zou, Z., 2016. Corrosion behavior of reheated CGHAZ of X80 pipeline steel in H2S-containing environments. Mater. Des. 99, 44-56. https://doi.org/10.1016/j.matdes.2016.03.036

\section{DEGRADAÇÃO E FORMAÇÃO DE PRODUTOS DE CORROSÃO NO AÇO API X65 EXPOSTO AOS MECANISMOS DE SWEET E SOUR CORROSION}

Souza, Rhuãn Costa (1) (rhuanmecufsj@gmail.com), Santos, Bernardo Augusto Farah (1) (bernardo.a.fs@hotmail.com), Sabará, Caio Vinícius Leão (1) (caio.leao@oi.com.br), Santos, Luan Carrera (1) (luancarrera777@gmail.com), Prachedes, Lucas Nascimento Santana(1) (lucasntst@gmail.com), Simões,

Thiago Araújo (2) (thiagoasimoes@gmail.com), Oliveira, Jefferson Rodrigues (3)

(jefferson.rodrigues@petrobras.com.br),Vaz, Gustavo Leitão(3) (gustavovaz@petrobras.com.br),Bueno, Alysson Helton Santos (1) (alyssonbueno@ufsj.edu.br)

(1) Universidade Federal de São João del Rei (UFSJ) - Dep. de eng. Mec.- 170, Centro, São João del-Rei, MG. (2) Universidade Federal da Paraíba (UFPB) - Cidade Universitária, João Pessoa, PB.

(3) Centro de Pesquisa da Petrobras (CENPES) - Av. Horácio Macedo, 950 - Cidade Universitária RJ.

ABSTRACT: The objective of this work was to study the electrochemical behavior and the formation of API $X 65$ carbon steel corrosion products when exposed to sweet and sour corrosion at the same time, knowing the competitive behavior of the $\mathrm{FeS}$ and $\mathrm{FeCO} 3$ formation. Due to the extreme toxicity and difficulty of working with the H2S, the use of Sodium Thiosulphate if shows an excellent alternative for H2S generation in metal/solution interface. EIS tests and mass Loss, were carried out in an autoclave with partial pressure of 5 bar of $\mathrm{CO} 2,25,90$ and $120^{\circ} \mathrm{C}$ with and without the presence of $\mathrm{H} 2 \mathrm{~S}$. The products formed were characterized by SEM and DRX. In conditions without $\mathrm{H} 2 \mathrm{~S}, 25^{\circ} \mathrm{C}$ no film formed on the samples, the $90^{\circ} \mathrm{C}$ a porous $\mathrm{FeCO} 3$ film formed on the material but the results he indicated a low protective capacity. Already $120^{\circ} \mathrm{C}$ the layer of $\mathrm{FeCO} 3$ graduated more stable and dense, reducing the corrosive processes. The addition of the thiosulfate ion proved efficient to simulate H2S effects, since accession of sour corrosion products were identified. FeS films formed reduced the corrosive effects of $\mathrm{CO} 2$ and hampered the $\mathrm{FeCO} 3$ precipitation in the material. The film competition was more intense at $120^{\circ} \mathrm{C}$, where the $\mathrm{FeCO} 3$ and $\mathrm{FeS}$ found stable formation conditions.

KEYWORDS: sweet/sour corrosion, EIE, weigth loss test, sodium thiosulfate.

IX COEN - Congresso de Engenharias da UFSJ Interconexão. 